\title{
Broadband PLC-channel equalisation in the frequency domain based on complementary sequences
}

ISSN 1751-8628

Received on 22nd September 2015

Revised on 26th April 2016

Accepted on 9th May 2016

doi: 10.1049/iet-com.2015.0924

www.ietdl.org

\author{
Sergio Moya ${ }^{1,2} \bowtie$, Matias Hadad ${ }^{2}$, Marcos Funes $^{2}$, Patricio Donato $^{2}$, Daniel Carrica $^{2}$ \\ ${ }^{1}$ Laboratorio de Electrónica, Universidad Nacional de Misiones, Facultad de Ingeniería, Oberá, Misiones, Argentina \\ ${ }^{2}$ Facultad de Ingeniería, Laboratorio de Instrumentación y Control (LIC), Instituto de Investigaciones Científicas y Tecnológicas en \\ Electrónica (ICYTE), Universidad Nacional de Mar del Plata, Av. Juan B. Justo 4302, Mar del Plata, Argentina \\ $\bowtie$ E-mail: sergio.e.moya@gmail.com
}

\begin{abstract}
Multipath channels, like power lines, have a periodic time-variant response that impacts on data transmission. Power Line Communications (PLC) performance, in the context of a cyclic-prefix single carrier modulation scheme, can benefit from frequency domain equalisation techniques. This study proposes a frequency-domain dynamic characterisation and equalisation algorithm based on the properties of complementary sequences (CSs). This proposal takes advantage of CS properties to reduce the complexity of the algorithm by performing all the operations in the frequency domain, and without the necessity of noise/variance estimators. The proposal is compared to some wellknown methods like zero forcing and minimum mean-square error (MMSE) through the transmission of data under different PLC channels. Bit error rate (BER) is also measured using Middleton Class A impulse noise (IN) model and the performance of all methods is finally evaluated under a time-variant PLC channel model showing the importance of dynamic equalisation on PLC systems. Reported computational resources and simulations show that the proposal is four time faster than MMSE and improves the BER performance by up to $4 \mathrm{~dB}$. In addition, the proposed identification algorithms shows to work properly even in PLC channels with attenuations higher than $40 \mathrm{~dB}$ and severe IN scenarios.
\end{abstract}

\section{Introduction}

Day after day, power line communications (PLCs) systems require higher data transmission rates to compete with established communication technologies such as digital subscriber lines or wireless services. Even though it is well known that power lines were not designed to provide a communication link, they remain as the most extended physical network. Low-voltage networks feature a vast tree-like topology with various length branches (of around $400 \mathrm{~m}$ long) terminated with variable loads of different impedances and characteristics [1]. Moreover, the broadband PLC (BB-PLC) channel presents a variable-time transmission medium with high interference due to the multiple reflections generated by the impedance mismatch and the non-linear behaviour of electrical devices connected to it $[2,3]$. As a consequence, transmitted signals suffer time-variable fading and inter-symbol interference (ISI) that requires a constantly dynamic compensation of the channel to reduce the transmission bit error rate (BER). Additionally, there is a high impulse noise (IN) present in the channel, which is notoriously different from the commonly assumed additive white Gaussian noise (AWGN) [4, 5]. Fortunately, this IN is well described by models such as Bernoulli-Gaussian or the Middleton Class A (MCA) [6]. The last one is supported by the results of many measurement campaigns [7], and is used in the present paper.

Considering this scenario, BB-PLC channel is a promising, but hostile medium to deliver information. Therefore, some techniques must be applied to reduce BER and offer further transmission speed and possibilities. In the case of ISI, a classical approach to mitigate it is to insert a cyclic prefix (CP) in the transmission data. This technique can be used with either single or multicarrier modulation schemes. However, the complexity and performance of single carrier (SC) modulation schemes such as CP-SC is comparable with those of a multicarrier [such as orthogonal frequency-division multiplexing (OFDM)] in the sense of a potential interoperability [8], but without its drawbacks, i.e. synchronisation issues or the peak-to-average ratio of OFDM transmitted signal [8-11].

Many SC frequency-domain equalisation (FDE) algorithms have been developed for wireless channels such as IEEE 802.15.3c, IEEE 802.11ad and those presented in [8]. It is argued that these methods can successfully deal with most general wireless channels, but, due to the fact that PLC-channels feature much more unfavourable characteristics such as fast-time variations and INs among others, to be discussed later, these algorithms may not be suitable for PLC systems. Traditional channel estimators are based on the minimisation of a cost function [12] or by using pilot/training symbols in order to compute the equaliser through decision-based tracking or adaptive filtering. These training symbols, with the objective of optimising the channel identification, should offer good spectral and correlation properties. Complementary sequences (CSs) present perfect autoand cross-correlation properties, and have been used for channel estimation in multiple-input-multiple-output systems [13], multi-path interference cancellation [14], wireless personal area network (WPAN) systems $[15,16]$ etc. These sequences, defined by Golay [17], have a distinctive property associated to the sum of their auto-correlation (AC) functions, which produces a Kronecker delta of amplitude $2 L$ ( $L$ being the sequences' length). This provides the channel impulse response (CIR) that can be used later to perform the equalisation.

Even though the use of CS presents good results to dynamically compensate channel variations, the equalisation algorithms $[15$, 16] usually are based on a time-domain channel estimation that requires substantial effort [8]. The present paper takes advantage of CS, but proposes less complex FDE algorithm performing all the calculations in the frequency domain. The developed algorithm is evaluated by simulating a complete CP-SC transmission scheme over several realistic and time-varying PLC-channel models yielding a BER reduction after the FDE procedure. These measurements were also made taking into consideration different configuration of MCA IN. Finally, the BER evolution of the 
proposed methods, in addition to their dynamic equalisation, is measured against time-varying PLC-channel model based on the three channels from [18]

\section{BB-PLC channels}

BB-PLC channels are bounded by the frequency band between 2 and $30 \mathrm{MHz}$. The power line comprises a large number of different conductors interconnected on a tree-like topology, with each extreme linked to an open-circuit or unknown time-variant impedances [19]. The main feature of BB-PLC channels is an average attenuation of about $20-30 \mathrm{~dB}$ even for short distances, and the presence of diverse deep-notches at random frequencies, where attenuation increases up to $30 \mathrm{~dB}$ [18]. In addition, the continuous switching of devices connected to the grid produces a wide variety of noise, but fundamentally, it generates a huge impedance mismatch, which entails serious reflection and multi-path effects $[4,20]$.

As mentioned earlier, wireless and PLC channels may be classified as being similar. Notwithstanding this, the later presents much more unfavourable characteristics. Both present path-loss and attenuations, but, in wireless cases, this behaviour is well known due to the extensive results reported in the literature. However, the PLC channels depend on the particular characteristics of the network topologies and cables, making path-loss and attenuations substantially more difficult to characterise [21]. Regarding time dispersion or root-mean-square delay spread, a comparison between wireless and PLC channels reports that PLC medium present delays even 1000 times larger than wireless cases, resulting in serious degradation on the frequency response, fundamentally on the phase behaviour of the channel [21, 22]. Time variation in PLC channels is another important factor worth mentioning. The continuous changes on impedance values at the end-points of the net produce fast variation of the frequency response for the PLC channel [21, 23]. The PLC channel can be considered invariant due to impedance variations in a period known as coherence time that is reported to be no smaller than $600 \mu \mathrm{s}[2,21]$. This time-variation highlights the importance of carrying out a pseudo-periodic compensation of the channel by measuring its BER: namely, when reported errors exceed a threshold, the system should update the equaliser to compensate the present channel.

In the present paper, simulations were performed using PLC channels proposed by Cañete et al. [18], which were devised considering all of these non-linearities and periodically

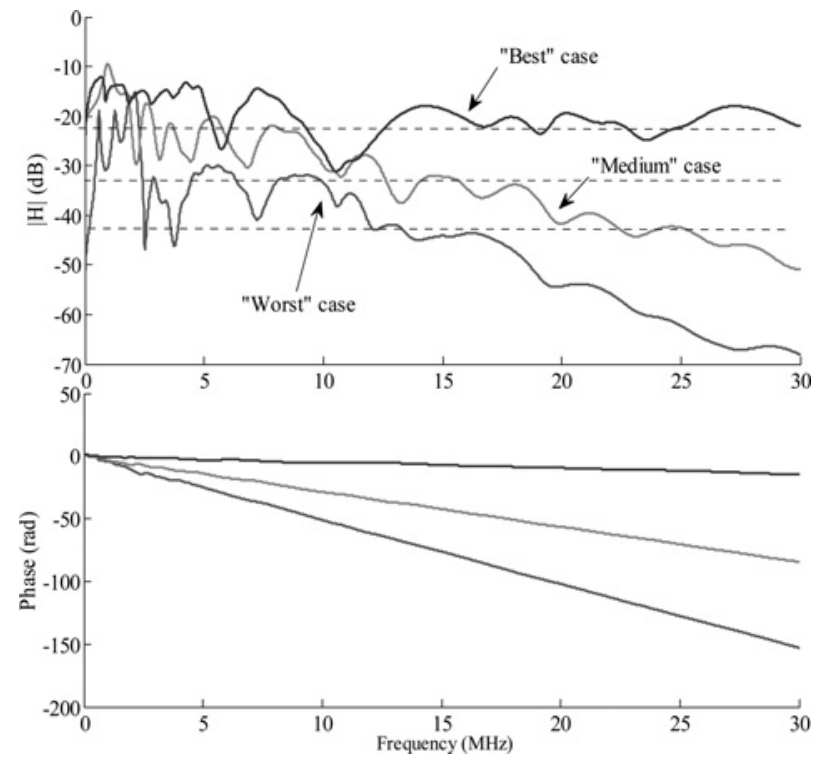

Fig. 1 BB-PLC channels. Magnitude (top) and phase (bottom) [18] time-varying characteristics. Fig. 1 shows magnitude and phase components of three different BB-PLC channels. These channels are classified as 'best', 'medium' and 'worst' depending on their magnitude and phase response. As shown with the horizontal black lines, for the best-case channel, the average attenuation produced is about $20 \mathrm{~dB}$, for the medium case it is about $35 \mathrm{~dB}$, and for the worst case it is higher than $40 \mathrm{~dB}$.

\section{Impulse noise model}

As in any other communication system, noise contributes to increase BER. Its spectrum is made up of coloured background noise, narrowband noise and IN. Coloured background and narrowband noises are the result of diverse low-power noise sources, so they are mainly considered as background noise [24]. IN is the most damaging component of PLC noise [5], and can be further categorised into three categories:

(i) Periodic IN synchronous to the main alternating current (AC) cycle is caused by switching rectifier diodes, which occurs synchronously with the mains cycle.

(ii) Periodic IN asynchronous to the main AC cycle, mostly caused by switching power supplies.

(iii) Asynchronous IN is caused by switching transients in the network, as the on/off switching of motors, drills etc.

The occurrence of these impulse events are mostly unpredictable in timing and severity. This phenomenon is modelled by different IN models such as the widely accepted MCA introduced in [6] and evaluated in [25]. The normalised complex probability density function (PDF) of MCA is given by

$$
p(z)=\sum_{m=0}^{\infty} I_{m} \frac{1}{\sqrt{2 \pi \sigma_{m}^{2}}} \exp \left(-\frac{z^{2}}{2 \sigma_{m}^{2}}\right)
$$

which represents a Gaussian PDF weighted by $I_{m}$, which is defined by

$$
I_{m}=\frac{\mathrm{e}^{-A} A^{m}}{m !}
$$

Equation (2), therefore, represents the density of impulse events in accordance with a Poisson distribution, where $A$ is the IN index and represents the density of impulse events in an observation period and

$$
\sigma_{m}^{2}=\sigma_{\mathrm{I}}^{2} \frac{m}{A}+\sigma_{\mathrm{G}}^{2}=\sigma_{\mathrm{G}}^{2}\left(\frac{m}{A \Gamma}+1\right)
$$

where $\sigma_{\mathrm{I}}^{2}$ represents the variance of the IN and $\sigma_{\mathrm{G}}^{2}$ is the variance of the AWGN background noise. In (3), $\Gamma$ is the Gaussian-to-impulse variance ratio defined as $\Gamma=\sigma_{\mathrm{G}}^{2} / \sigma_{\mathrm{I}}^{2}$.

\section{CP-SC system description}

To deal with the worst BB-PLC scenarios, it is necessary to dynamically estimate and equalise the channel taking into account noise and time variations. This section introduces the dynamic CP-SC FDE scheme used in this paper (Fig. 2). Data flow in Fig. 2 starts by mapping symbols made up of 'data in' information bits, and then by performing a serial-to-parallel (S/P) conversion.

Supposing the transmission of $l$ data blocks $\boldsymbol{d}$, composed of $M$ information bits with variance $\sigma_{d}^{2}$

$$
\boldsymbol{d}_{M}^{(l)}=\left[d_{0}^{(l)}, d_{1}^{(l)}, d_{2}^{(l)}, \ldots, d_{M-1}^{(l)}\right]^{\mathrm{T}}
$$

FDE can be conducted if the transmitted signal forces the linear convolution with the CIR to be circular, and this occurs when data 


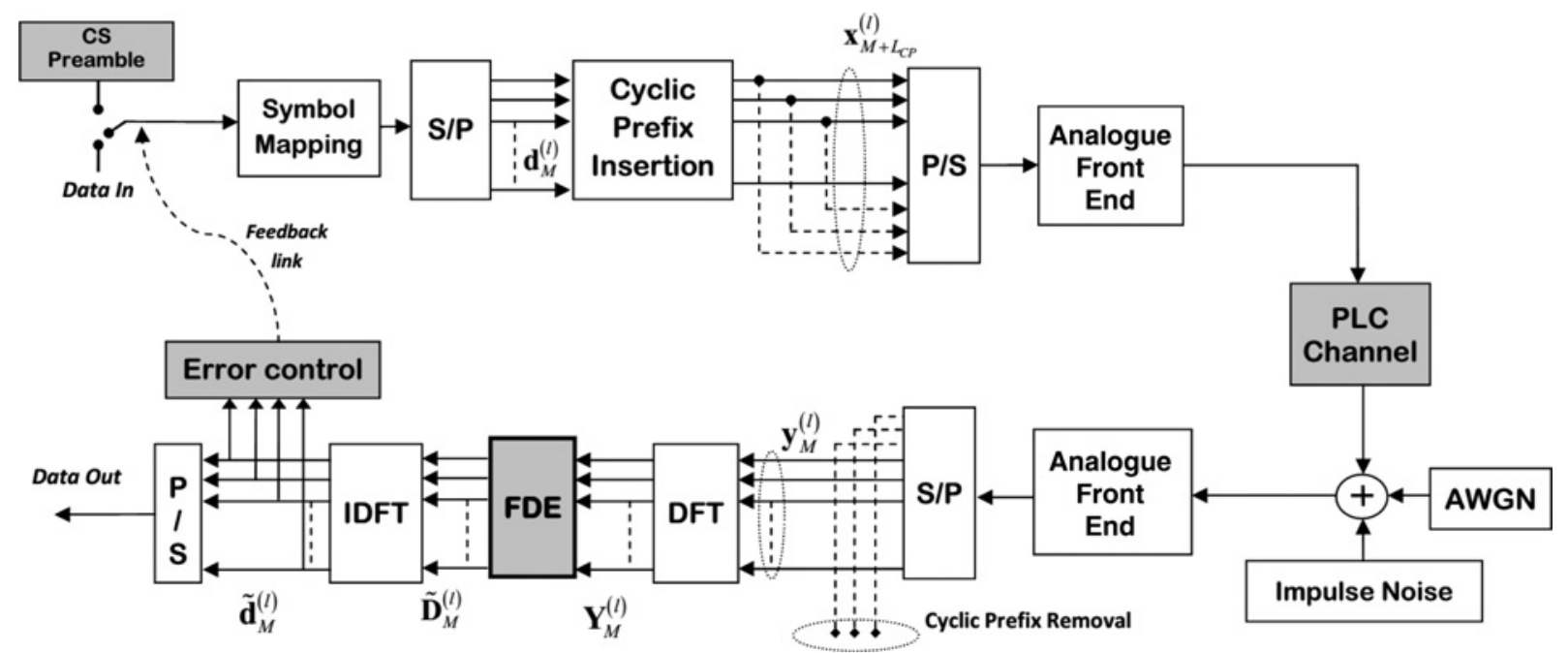

Fig. 2 CP-SC communication system with FDE

blocks are cyclically extended by the copy of the last $L_{\mathrm{CP}}$ symbols [10]

$$
\boldsymbol{x}_{M+L_{\mathrm{cp}}}^{(l)}=\left[d_{M-L_{\mathrm{CP}}}^{(l)}, \ldots, d_{M-1}^{(l)}, d_{0}^{(l)}, \ldots, d_{M-1}^{(l)}\right]^{\mathrm{T}}
$$

$\boldsymbol{x}_{M+L_{\mathrm{cp}}}^{(l)}$ is composed of $M+L_{\mathrm{CP}}$ length blocks which are converted into serial bits by a parallel-to-serial converter, and transmitted through the PLC channel using an analogue front-end (AFE) device. CIR, of length $L_{\mathrm{CIR}}$, is given by

$$
\boldsymbol{h}_{L_{\mathrm{CIR}}}^{(l)}=\left[h_{0}^{(l)}, h_{1}^{(l)}, h_{2}^{(l)}, \ldots, h_{L_{\mathrm{CIR}}-1}^{(l)}\right]^{\mathrm{T}}
$$

As described in Section 3, PLC noise is predominantly made up of impulse and background noises. However, in order to obtain a simplified model, suppose the addition of ideal AWGN of variance $\sigma_{\mathrm{v}}^{2}$

$$
\boldsymbol{v}_{M}^{(l)}=\left[v_{0}^{(l)}, v_{1}^{(l)}, v_{2}^{(l)}, \ldots, v_{M-1}^{(l)}\right]^{\mathrm{T}}
$$

In the receiver, the input signal is discretised by the AFE and converted into parallel by an $\mathrm{S} / \mathrm{P}$ converter. After that, $\mathrm{CP}$ containing interference from previous blocks is removed, leaving blocks $\boldsymbol{y}_{M}^{(l)}$ of length $M$ with no distortion, insofar as $L_{\mathrm{CP}}>L_{\mathrm{CIR}}$.

The received signal $\boldsymbol{y}_{M}^{(l)}=\left[y_{0}^{(l)}, y_{1}^{(l)}, y_{2}^{(l)}, \ldots, y_{M-1}^{(l)}\right]^{\mathrm{T}}$ can be expressed in a matrix notation as follows [26]

$$
\boldsymbol{Y}_{M}^{(l)}=\boldsymbol{H}_{M}^{(l)} \boldsymbol{D}_{M}^{(l)}+\boldsymbol{V}_{M}^{(l)}
$$

where $\boldsymbol{H}_{M}^{(l)}=\operatorname{diag}\left(\mathbf{D F T}_{M}\left[\boldsymbol{h}_{L_{\mathrm{CIR}}^{(l)}}\right]\right), \boldsymbol{D}_{M}^{(l)}=\mathbf{D F T}_{M}\left[\boldsymbol{d}_{M}^{(l)}\right]$ is the $l$ th block of transmitted symbols and $\boldsymbol{V}_{M}^{(l)}=\mathbf{D F T}\left[\boldsymbol{v}_{M}^{(l)}\right]$ is the overall independent Gaussian noise vector present on the PLC channel. Here, $\mathbf{D F T}_{M}$ denotes the $M$-point discrete Fourier Transform and $\operatorname{diag}(\cdot)$ is the diagonal matrix with the elements of $(\cdot)$ along the diagonal.

Fig. 2 also shows an error control and feedback link between the receiver and the transmitter. This step is necessary in order to perform a dynamic compensation of the channel in time-varying systems.

\section{Frequency-domain equalisation}

Several basic techniques are used to estimate PLC channels in SC frequency-domain systems: namely, to obtain $\boldsymbol{H}_{M}^{(l)}$ matrix from (8). The most basic is known as zero forcing (ZF), which consists in the multiplication of the received vector data by the inverse of the channel response.

From (8), it can be seen that the channel distortion can be perfectly compensated by pre-multiplying $\boldsymbol{Y}_{M}^{(l)}$ by the inverse of $\boldsymbol{H}_{M}^{(l)}$

$$
\begin{gathered}
\left(\boldsymbol{H}_{M}^{(l)}\right)^{-1} \boldsymbol{Y}_{M}^{(l)}=\left[\left(\boldsymbol{H}_{M}^{(l)}\right)^{-1} \boldsymbol{H}_{M}^{(l)}\right] \boldsymbol{D}_{M}^{(l)}+\left(\boldsymbol{H}_{M}^{(l)}\right)^{-1} \boldsymbol{V}_{M}^{(l)} \\
\mathrm{ZF} \\
\boldsymbol{W}_{M}^{(l)} \boldsymbol{Y}_{M}^{(l)}=\boldsymbol{D}_{M}^{(l)}+\frac{\boldsymbol{V}_{M}^{(l)}}{\boldsymbol{H}_{M}^{(l)}}
\end{gathered}
$$

where

$$
\stackrel{\mathrm{ZF}}{\boldsymbol{W}_{M}^{(i)}}=\frac{1}{\boldsymbol{H}_{M}^{(l)}}
$$

and $i$ represents the current $i$ th equalisation term. ZF strategy is valid only when the channel noise is negligible and CIR is ideally known, obtaining

$$
\mathbf{D F T}^{-1}\left[\begin{array}{l}
\mathrm{ZF} \\
\boldsymbol{W}_{M}^{(i)}
\end{array} \boldsymbol{Y}_{M}^{(l)}\right]=\mathbf{D F T}^{-1}\left[\boldsymbol{D}_{M}^{(l)}\right]=\boldsymbol{d}_{M}^{(l)}
$$

The main drawback of the $\mathrm{ZF}$ technique is the excessive noise enhancement evidenced by the last term of (10). This enhancement is particularly relevant when $\boldsymbol{H}_{M}^{(l)}$ has spectral nulls, or when the average attenuation of the PLC channel is big, i.e. more than $20 \mathrm{~dB}$. For this reason, another equalisation strategy, which includes channel noise, is used. On this strategy, based on the minimum mean-square error (MMSE) criteria, the equaliser term takes the form of [9]

$$
\underset{\boldsymbol{W}_{M}^{(i)}}{\operatorname{MMSE}}=\frac{\overline{\boldsymbol{H}_{M}^{(l)}}}{\left|\boldsymbol{H}_{M}^{(l)}\right|^{2}+\left(\sigma_{\mathrm{v}}^{2} / \sigma_{d}^{2}\right)}
$$

where $\overline{(\cdot)}$ denotes the complex conjugate. The additive term $\sigma_{\mathrm{v}}^{2} / \sigma_{d}^{2}$ MMSE

prevents $\boldsymbol{W}_{M}^{(i)}$ from taking infinite values in the case of spectral 
nulls of $\boldsymbol{H}_{M}^{(l)}$; consequently, the MMSE equaliser can be used for all types of channels [9].

\section{FDE using CSs}

The challenge of obtaining a precise and robust estimation of $\boldsymbol{H}_{M}^{(l)}$ can be achieved fully through the use of CSs. These sequences are defined as two binary sequences, $\boldsymbol{a}_{\boldsymbol{L}}[k]$ and $\boldsymbol{b}_{\boldsymbol{L}}[k]$, where $k$ represents the $k$ th element of the sequence, whose length is $L=2^{n}$, $(n=1,2, \ldots, L)$. The AC function of each sequence is

$$
\begin{aligned}
& \boldsymbol{C}_{\boldsymbol{a}_{\boldsymbol{L}} \boldsymbol{a}_{\boldsymbol{L}}}[\boldsymbol{i}]=\sum_{k=1}^{k=L} a_{L}[k] \cdot a_{L}[k+i] \\
& \boldsymbol{C}_{\boldsymbol{b}_{\boldsymbol{L}} \boldsymbol{b}_{\boldsymbol{L}}}[\boldsymbol{i}]=\sum_{k=1}^{k=L} b_{L}[k] \cdot b_{L}[k+i]
\end{aligned}
$$

The pairs of CSs are characterised by their sum of ACs

$$
\boldsymbol{C}_{a_{L} a_{L}}[\boldsymbol{i}]+\boldsymbol{C}_{\boldsymbol{b}_{L} \boldsymbol{b}_{L}}[\boldsymbol{i}]= \begin{cases}0, & i \neq 0 \\ 2 L, & i=0\end{cases}
$$

which is a Kronecker delta of amplitude $2 L$. In frequency domain, this Kronecker delta can be seen as a constant frequency response, and the aforesaid analysis can be considered as

$$
\begin{aligned}
& A_{L}=\mathbf{D F T}_{L}\left[a_{L}[k]\right] \\
& B_{L}=\mathbf{D F T}_{L}\left[b_{L}[k]\right]
\end{aligned}
$$

In this case, the $\mathrm{AC}$ becomes

$$
\begin{aligned}
& C_{L}^{A A}=\boldsymbol{A}_{\boldsymbol{L}} \overline{\boldsymbol{A}_{\boldsymbol{L}}} \\
& C_{L}^{B B}=\boldsymbol{B}_{\boldsymbol{L}} \overline{\boldsymbol{B}_{\boldsymbol{L}}}
\end{aligned}
$$

where $\overline{A_{L}}$ represents the complex conjugate of $A_{L}$. Equation (15) becomes

$$
C_{L}^{A A}+C_{L}^{B B}=2 L
$$

Suppose a frequency selective channel spectrum represented by its impulse response $h$ and its $L$-sample frequency response $H_{L}$. If $A_{L}$ and $B_{L}$ are sent through $H_{L}$, the received sequences become $A_{L}^{\prime}+V_{L}^{1}$ and $B_{L}^{\prime}+V_{L}^{2}$, respectively, where $V_{L}^{1}$ and $V_{L}^{2}$ are independent frequency-domain noise vectors, and (17) can change into

$$
C_{L}^{A^{\prime} A}+C_{L}^{B^{\prime} B}=\left(A_{L}^{\prime}+V_{L}^{I}\right) \overline{A_{L}}+\left(B_{L}^{\prime}+V_{L}^{2}\right) \overline{B_{L}}
$$

Since $A_{L}^{\prime}=H_{L} A_{L}$ and $B_{L}^{\prime}=H_{L} B_{L}$

$$
\begin{aligned}
C_{L}^{A^{\prime} A}+C_{L}^{B^{\prime} B} & =\left(H_{L} A_{L}+V_{L}^{1}\right) \overline{A_{L}}+\left(H_{L} B_{L}+V_{L}^{2}\right) \overline{B_{L}} \\
& =H_{L}\left(A_{L} \overline{A_{L}}+B_{L} \overline{B_{L}}\right)+\overline{A_{L}} V_{L}^{1}+\overline{B_{L}} V_{L}^{2} \\
& =(2 L) H_{L}+V_{L}=H_{L}^{\prime}
\end{aligned}
$$

which is the frequency response of the channel that distorts both sequences, multiplied by the gain-process $2 L$ plus a correlated noise vector $\boldsymbol{V}_{L}$. By using $H_{L}^{\prime}$ term, the $\mathrm{ZF}$ equalisation term based on CS (CS-ZF) could be defined as

$$
\stackrel{C S-\mathbf{Z F}_{L}^{(i)}}{\boldsymbol{W}_{L}}=\frac{2 \boldsymbol{L}}{\boldsymbol{H}_{\boldsymbol{L}}^{\prime}}
$$

and the CS-MMSE equalisation term can be calculated by using (13) though taking into account that $H_{L}^{\prime}$ is obtained by the procedure indicated in (20)

$$
\stackrel{\boldsymbol{W}_{L}^{(i)}}{\text { CS-MSE }}=\frac{\overline{\boldsymbol{H}_{\boldsymbol{L}}^{\prime}}}{\left|\boldsymbol{H}_{\boldsymbol{L}}^{\prime}\right|^{2}+\left(\boldsymbol{\sigma}_{\mathrm{v}}^{2} / \boldsymbol{\sigma}_{\mathrm{d}}^{2}\right)}
$$

The major disadvantage of MMSE-based equalisation methods is that they demand the use of some noise/variance-estimation process to calculate the denominator of (22). This process can be avoided by using CS and least-squares (LS) minimisation technique in order to obtain an LS-based equalisation term.

As shown in (9), the basic idea of FDE is to multiply the received signals $\boldsymbol{Y}_{M}^{(l)}$ by an equalisation term $\boldsymbol{W}_{M}^{(i)}$ while trying to avoid the unnecessary noise enhancement, fundamentally in those sectors of the spectrum that contain minimums or spectral nulls. With respect to the scheme shown in Fig. 2

$$
\tilde{\boldsymbol{D}}_{M}^{(l)}=\boldsymbol{W}_{M}^{(i)} \boldsymbol{Y}_{M}^{(l)}
$$

where $\tilde{\boldsymbol{D}}_{M}^{(l)}$ denotes the estimation of $\boldsymbol{D}_{M}^{(l)}$ (Fig. 2). The equalisation term $\boldsymbol{W}_{M}^{(i)}$ can be computed by minimising the quadratic error $(e)$ between the transmitted and the equalised signals

$$
\begin{aligned}
e^{i} & =\sum_{M}\left|\boldsymbol{D}_{M}^{(l)}-\boldsymbol{W}_{M}^{(i)} \boldsymbol{Y}_{M}^{(l)}\right|^{2}=\sum_{k}\left|\boldsymbol{D}_{M}^{(l)}-\tilde{\boldsymbol{D}}_{M}^{(l)}\right|^{2} \\
& =\sum_{M}\left(\boldsymbol{D}_{M}^{(l)}-\boldsymbol{W}_{M}^{(i)} \boldsymbol{Y}_{M}^{(l)}\right) \overline{\left(\boldsymbol{D}_{M}^{(l)}-\boldsymbol{W}_{M}^{(i)} \boldsymbol{Y}_{M}^{(l)}\right)}
\end{aligned}
$$

In this sense, $\boldsymbol{W}_{M}^{(i)}$ can be obtained at the receiver transmitting as $\boldsymbol{D}_{M}^{(l)}$ a preamble composed by consecutive CSs of length $L$. Assuming that $L=M$, the spectrum of each transmitted sequence is modified by the channel, resulting in the sequences spectra $A_{M}^{\prime}$ and $B_{M}^{\prime}$ These two received sequences can be introduced into (24), resulting in

$$
e=\sum_{M}\left[\begin{array}{c}
\left(\boldsymbol{A}_{\boldsymbol{M}}-\boldsymbol{W}_{\boldsymbol{M}} A_{M}^{\prime}\right)\left(\overline{\boldsymbol{A}_{\boldsymbol{M}}-\boldsymbol{W}_{\boldsymbol{M}} A_{M}^{\prime}}\right) \\
+\left(\boldsymbol{B}_{\boldsymbol{M}}-\boldsymbol{W}_{\boldsymbol{M}} B_{M}^{\prime}\right)\left(\overline{\boldsymbol{B}_{M}-\boldsymbol{W}_{\boldsymbol{M}} B_{M}^{\prime}}\right)
\end{array}\right]
$$

and operating

$$
e=\sum_{M}\left(\begin{array}{c}
\left|\boldsymbol{A}_{\boldsymbol{M}}\right|^{2}+\left|\boldsymbol{B}_{\boldsymbol{M}}\right|^{2}+\left|\boldsymbol{W}_{\boldsymbol{M}}\right|^{2}\left|A_{M}^{\prime}\right|^{2}+\left|\boldsymbol{W}_{\boldsymbol{M}}\right|^{2}\left|B_{M}^{\prime}\right|^{2} \\
-2 \operatorname{Re}\left\{\boldsymbol{W}_{\boldsymbol{M}} A_{M}^{\prime} \overline{\boldsymbol{A}_{\boldsymbol{M}}}\right\}-2 \operatorname{Re}\left\{\boldsymbol{W}_{\boldsymbol{M}} B_{M}^{\prime} \overline{\boldsymbol{B}_{\boldsymbol{M}}}\right\}
\end{array}\right)
$$

Then, splitting $\boldsymbol{W}_{\boldsymbol{M}}$ in its real and imaginary parts $\left(\boldsymbol{W}_{\mathbf{R e}}, \boldsymbol{W}_{\mathbf{I m}}\right)$

$$
e=\sum_{M}\left(\begin{array}{c}
\left|A_{M}\right|^{2}+\left|B_{M}\right|^{2}+W_{\mathrm{Re}}^{2}\left(\left|A_{M}^{\prime}\right|^{2}+\left|B_{M}^{\prime}\right|^{2}\right) \\
+W_{\operatorname{Im}}^{2}\left(\left|A_{M}^{\prime}\right|^{2}+\left|B_{M}^{\prime}\right|^{2}\right) \\
-2 \boldsymbol{W}_{\operatorname{Re}} \operatorname{Re}\left\{A_{M}^{\prime} \overline{A_{M}}+B_{M}^{\prime} \overline{B_{M}}\right\} \\
-2 \boldsymbol{W}_{\operatorname{Im}} \operatorname{Im}\left\{A_{M}^{\prime} \overline{A_{M}}+B_{M}^{\prime} \overline{B_{M}}\right\}
\end{array}\right)
$$

Minimising $e$, in relation to $\boldsymbol{W}_{\mathbf{R e}}$ and $\boldsymbol{W}_{\mathbf{I m}}$, respectively

$$
\begin{gathered}
\frac{\partial e}{\partial \boldsymbol{W}_{\mathbf{R e}}}=2\left(\boldsymbol{W}_{\mathbf{R e}}\left(\left|A_{M}^{\prime}\right|^{2}+\left|B_{M}^{\prime}\right|^{2}\right)-\operatorname{Re}\left\{A_{M}^{\prime} \overline{A_{M}}+B_{M}^{\prime} \overline{B_{M}}\right\}\right)=0 \\
\frac{\partial e}{\partial \boldsymbol{W}_{\mathbf{I m}}}=2\left(\boldsymbol{W}_{\mathbf{I m}}\left(\left|A_{M}^{\prime}\right|^{2}+\left|B_{M}^{\prime}\right|^{2}\right)+\operatorname{Im}\left\{A_{M}^{\prime} \overline{A_{M}}+B_{M}^{\prime} \overline{B_{M}}\right\}\right)=0
\end{gathered}
$$


which yields

$$
\begin{aligned}
\boldsymbol{W}_{\mathbf{R e}} & =\frac{\operatorname{Re}\left\{A_{M}^{\prime} \overline{A_{M}}+B_{M}^{\prime} \overline{B_{M}}\right\}}{\left|A_{M}^{\prime}\right|^{2}+\left|B_{M}^{\prime}\right|^{2}} \\
\boldsymbol{W}_{\mathbf{I m}} & =\frac{-\operatorname{Im}\left\{A_{M}^{\prime} \overline{A_{M}}+B_{M}^{\prime} \overline{B_{M}}\right\}}{\left|A_{M}^{\prime}\right|^{2}+\left|B_{M}^{\prime}\right|^{2}}
\end{aligned}
$$

Building $\boldsymbol{W}_{\boldsymbol{M}}$ from (29)

$$
\boldsymbol{W}_{\boldsymbol{M}}=\frac{\operatorname{Re}\left\{A_{M}^{\prime} \overline{A_{M}}+B_{M}^{\prime} \overline{B_{M}}\right\}-\mathrm{j} \operatorname{Im}\left\{A_{M}^{\prime} \overline{A_{M}}+B_{M}^{\prime} \overline{B_{M}}\right\}}{A_{M}^{\prime} \overline{A_{M}^{\prime}}+B_{M}^{\prime} \overline{B_{M}^{\prime}}}
$$

with

$$
\begin{aligned}
& \operatorname{Re}\left\{A_{M}^{\prime} \overline{A_{M}}\right\}-\mathrm{j} \operatorname{Im}\left\{A_{M}^{\prime} \overline{A_{M}}\right\}=\overline{A_{M}^{\prime} \overline{A_{M}}}=\overline{A_{M}^{\prime}} A_{M} \\
& \operatorname{Re}\left\{B_{M}^{\prime} \overline{B_{M}}\right\}-\mathrm{j} \operatorname{Im}\left\{B_{M}^{\prime} \overline{B_{M}}\right\}=\overline{B_{M}^{\prime} \overline{B_{M}}}=\overline{B_{M}^{\prime}} B_{M}
\end{aligned}
$$

Then, the CS-LS-based equalisation term is

$$
\boldsymbol{W}_{L}^{\text {CS-LS }}=\frac{\overline{A_{M}^{\prime}} A_{M}+\overline{B_{M}^{\prime}} B_{M}}{A_{M}^{\prime} \overline{A_{M}^{\prime}}+B_{M}^{\prime} \overline{B_{M}^{\prime}}}
$$

If CSs are sent periodically, with a period less than or equal to the coherence-channel time, the equaliser compensates the received data by performing (23) in the frequency domain and without the need of making a separate noise estimation.

After the equalisation, the PLC system continues the data processing by performing the inverse DFT and symbol demapping, as it is shown in Fig. 3, which illustrates a summarised scheme of the proposed equalisation algorithm.

In this scheme, time-domain packages $\boldsymbol{d}_{M}^{(l)}$, headed by a preamble made up of time-domain CS $a_{L}[k]$ and $b_{L}[k]$ are sent through the PLC channel. An $M$-size DFT block is used to convert time-domain signals into frequency domain. Channel estimation starts with switch $(\mathrm{SW})=1$, enabling the storage of the received preamble, now comprising $\boldsymbol{A}_{M}^{\prime}$ and $\boldsymbol{B}_{M}^{\prime}$ in a $2 M$-sizes register. With both sequences available, the equalisation term $\boldsymbol{W}_{L}^{(i)}$ can be computed and CS-LS

registered. Once $\boldsymbol{W}_{L}^{(i)}$ is registered, the switch enables FDE (SW = 2) until the channel changes again.

\subsection{Computational resources}

Computational resources associated with the three channel estimation and equalisation methods presented in this section are reviewed below. CS-ZF method (21) requires two complex multiplications, one complex sum and one complex division. On the other hand, obtaining the CS-MMSE (22) term demands $H_{L}^{\prime}$ from CS-ZF, so it requires two complex multiplications, one real division, one complex division, one real sum, one complex absolute value and two real-time variance calculations. Finally, the

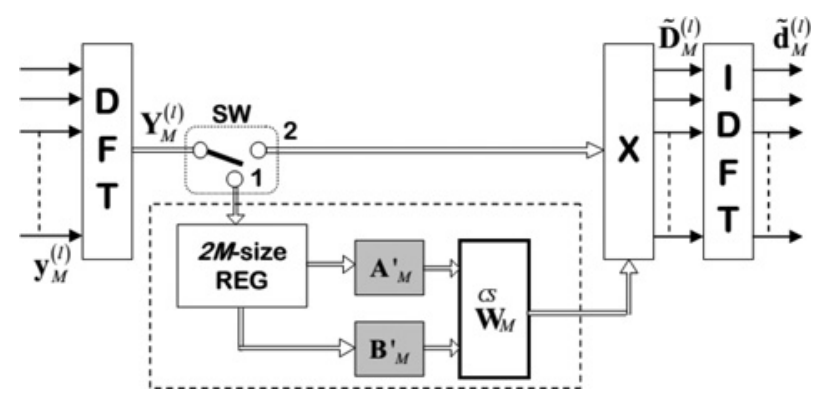

Fig. 3 Proposed equalisation scheme
Table 1 Computational resources

\begin{tabular}{lccccc}
\hline & $\begin{array}{c}\text { Real } \\
\text { sums }\end{array}$ & $\begin{array}{c}\text { Real } \\
\text { multiplications }\end{array}$ & $\begin{array}{c}\text { Real } \\
\text { divisions }\end{array}$ & $\begin{array}{c}\text { Real-time } \\
\text { variance }\end{array}$ & $\begin{array}{c}\text { Time, } \\
\mathrm{ms}\end{array}$ \\
\hline CS-ZF & 9 & 14 & 1 & - & 0.072 \\
CS-MMSE & 9 & 16 & 2 & 2 & 0.496 \\
CS-LS & 12 & 16 & 2 & - & 0.121 \\
\hline
\end{tabular}

proposed CS-LS method (32) demands only four complex multiplications, two complex sums and two real divisions. For ease the comparison, the previous operations are expressed in real mathematical operations, summarised in Table 1.

It should be noted that real-time variance calculations involve several sums and multiplications, but that will vary depending on the implementation. Since each method uses different amounts of different operations, the computational effort of the methods is compared using their required execution times. The methods were implemented in MATLAB and the presented result is the average required time of 1000 runs of each method. From the results, it can be seen that the proposed CS-LS method is four times faster than CS-MMSE, but 1.68 times slower than CS-ZF.

\section{Simulation results}

The proposed equalisation methods are compared with ideal ZF and MMSE classic methods. In such ideal methods, the channel is considered to be absolutely known and stable, and the equalisation taps are computed based on (11) and (13), respectively. CS-based methods, on the other hand, need to estimate the channel by using the received signal. As previously mentioned, BB-PLC noise differs significantly from the theoretical AWGN, so, in order to simulate the CP-SC equalisation methods in a more realistic environment, simulations are unfolded into:

- BER performance of all methods using AWGN under three time-fixed BB-PLC multi-path channels with low-pass profile and attenuations starting from $20 \mathrm{~dB}$ (Fig. 1).

- BER provided by the proposed methods through the same three channels (Fig. 1), but under MCA IN with different configurations. IN interferes on channel estimation process by distorting the CS, therefore, ideal ZF and MMSE methods cannot be assessed because they do not perform any channel estimation.

- Time evolution of BER and dynamic equalisation under time-variable PLC-channel model. This is done by using channels from Fig. 1 with a specific algorithm that simulates realistic time variations.

All simulations were performed using an SC-binary phase shift keying (BPSK) modulation with a carrier frequency of $11 \mathrm{MHz}$, a bandwidth of $10 \mathrm{MHz}$ and several signal-to-noise ratios (SNRs).

\subsection{BER performance with AWGN}

Fig. 4 depicts the BER from all the evaluated methods with AWGN. It is important to underline that the term $\boldsymbol{H}_{M}^{(l)}$ for (11) and (13) (namely, ideal ZF and MMSE) represents the CIR, and therefore as mentioned above, this produces, for the specific case of $\mathrm{ZF}$, a performance reduction in those sectors of the channel that possess high attenuation or deep-notches (10).

For the 'best' case (Fig. 4a), it should be noted the overall good performance of the proposed CS-based estimation and equalisation methods against ideal ZF and MMSE. The best performances were obtained by CS-LS and ideal MMSE, which, in this case, performs similarly in most of the SNR range (zoom of Fig. 4a). Between the CS-based methods, CS-LS clearly produces the best results, and CS-MMSE exceeds CS-ZF. As can be seen in Fig. 1, for the carrier frequency of $11 \mathrm{MHz}$, the 'best' channel presents a notch, reducing the performance on those methods based on the ZF technique. Fig. $4 a$ also shows that the BER obtained when the 

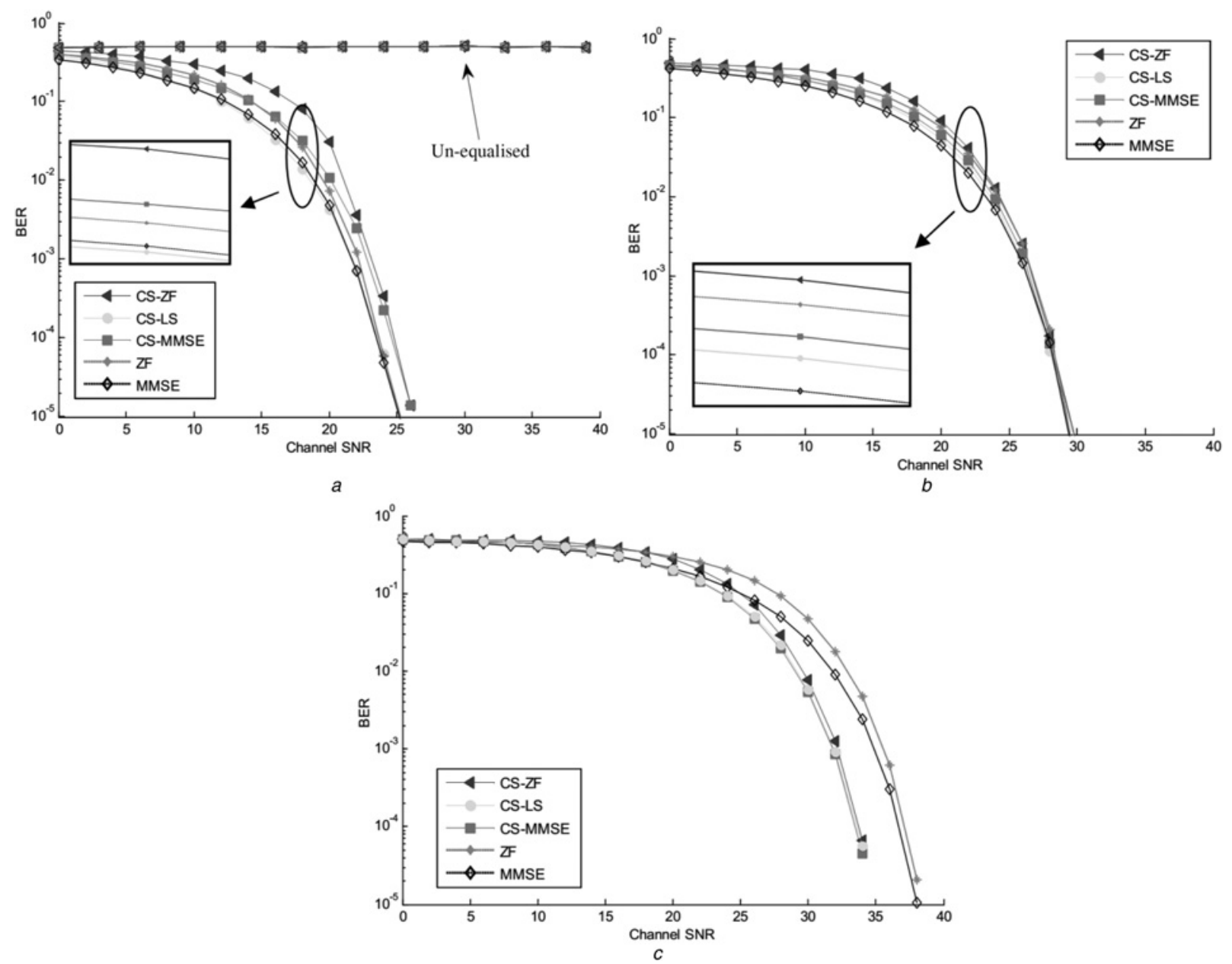

Fig. 4 BER over three PLC channels with AWGN

$a$ BER over 'best' PLC channel with AWGN

$b$ BER over 'medium' PLC channel with AWGN

$c$ BER over 'worst' PLC channel

estimation and equalisation process is not used is about 0.5 , rendering the use of an equalisation method necessary in BB-PLC communication systems.
With regard to the 'medium' case PLC channel depicted in Fig. $4 b$, all evaluated methods appear to work similarly. However, the zoom on Fig. $4 b$ shows that for the medium channel, ideal

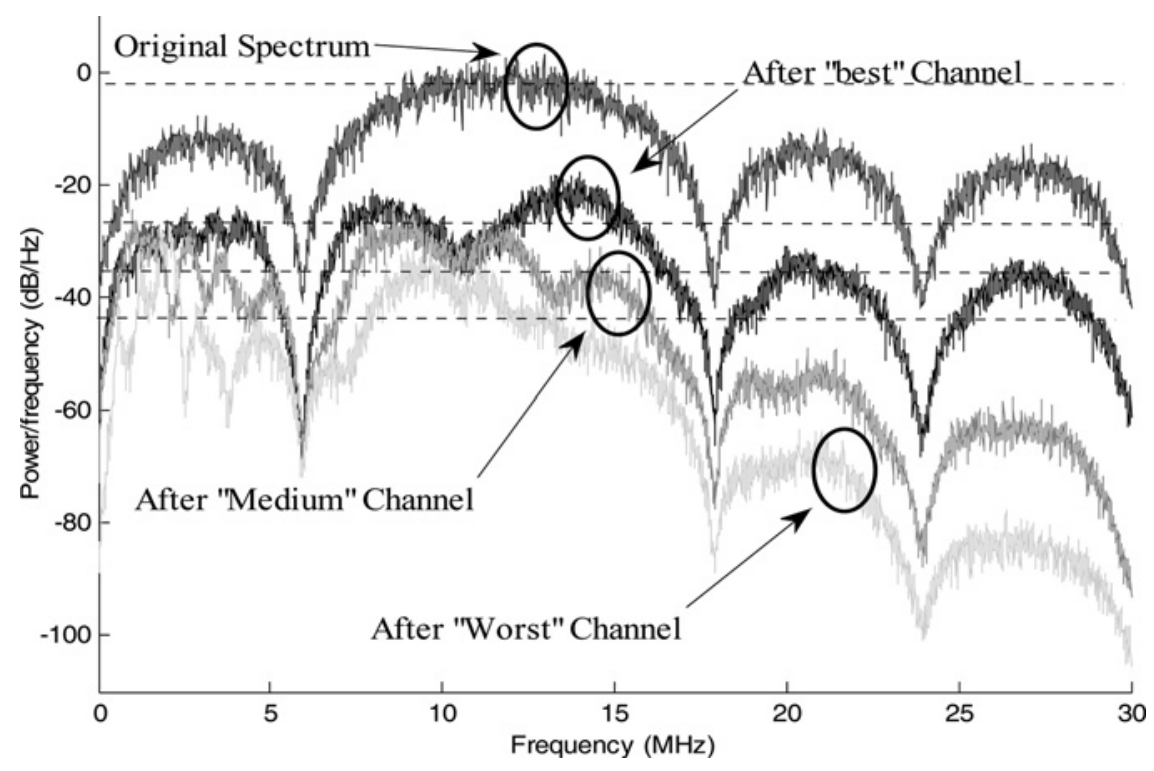

Fig. 5 SC-BPSK power spectrum 


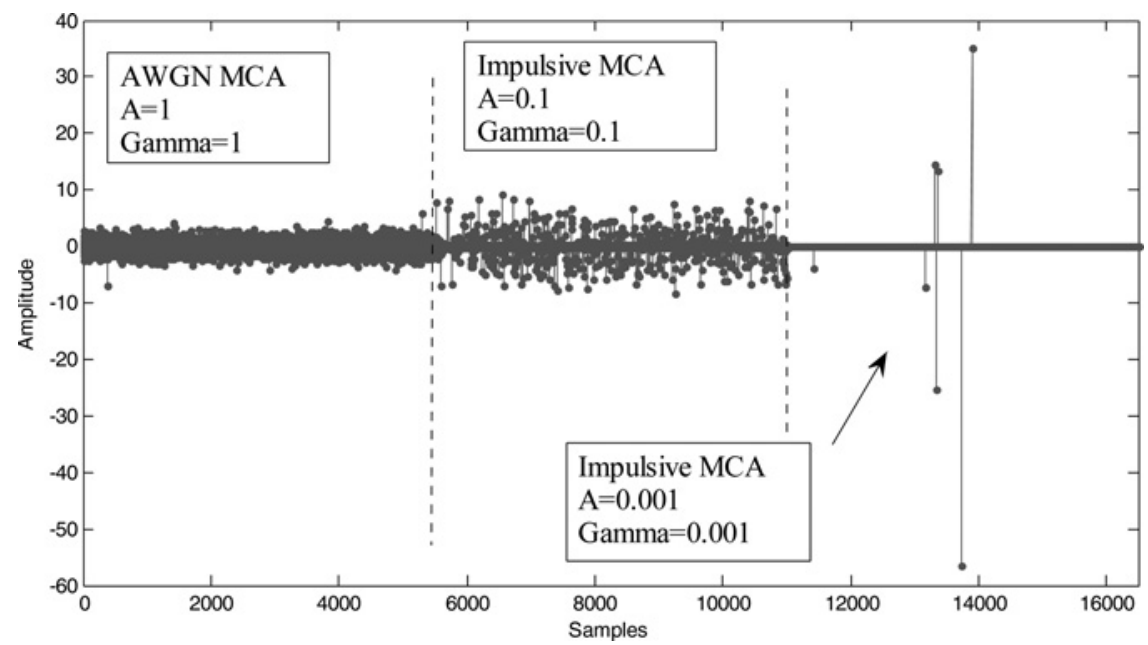

Fig. 6 MCA IN profiles used in simulations

MMSE produces best results, followed by CS-LS and CS-MMSE. Once again, a small notch in the channel, centred in the carrier frequency, and an average attenuation of $30 \mathrm{~dB}$ in the transmitted bandwidth make both MMSE methods and the proposed LS the most suitable methods for this type of channel. By comparing Fig. $4 a$ with Fig. $4 b$, it should be noted that the 'medium' channel produces a loss of about $5 \mathrm{~dB}$ in all evaluated methods, owing that to the attenuation and phase introduced by the channel on the used bandwidth (Fig. 1).

Finally, for the worst scenario, as shown in Fig. $4 c$, ideal ZF/ MMSE methods compromise their performance in $\sim 7-9 \mathrm{~dB}$ with respect to Fig. $4 b$ and begin to be overtaken by CS-based
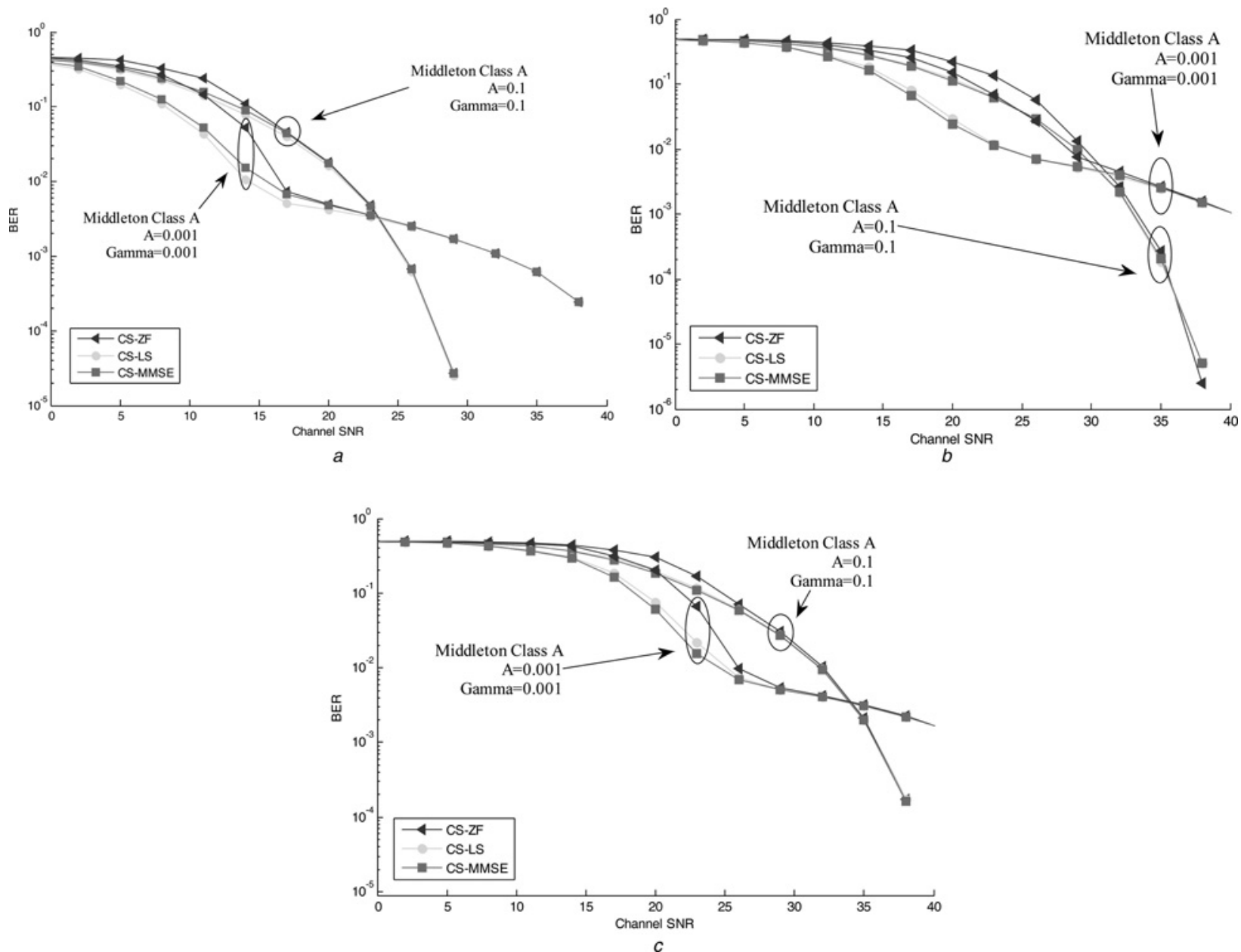

Fig. 7 BER over three PLC channels with IN

$a$ BER over 'best' PLC channel with IN

$b$ BER over 'medium' PLC channel with IN

$c$ BER over 'worst' PLC channel with IN 

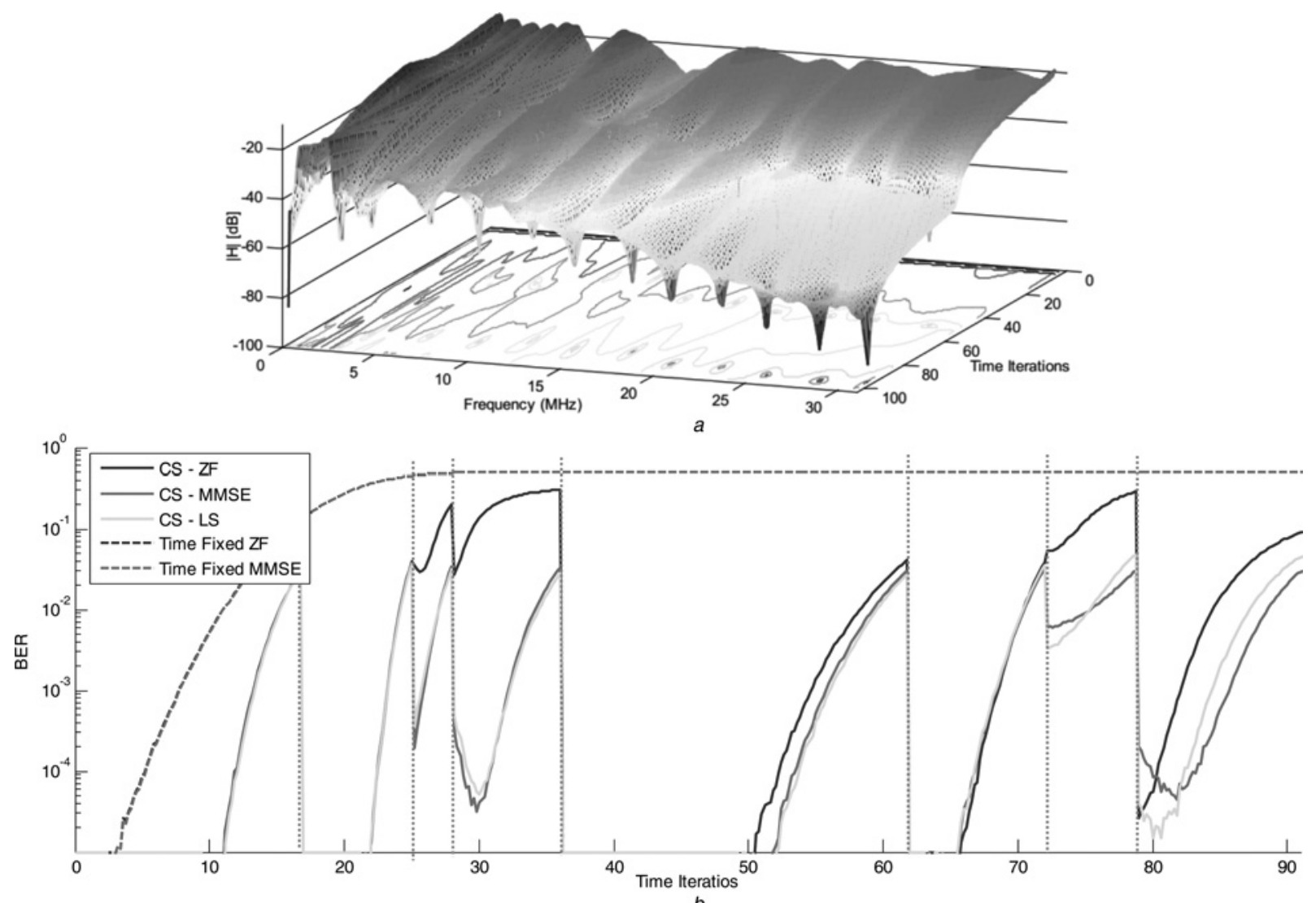

Fig. 8 Evaluation of equalisation proposal in time-varying PLC scenario

$a$ Time-varying PLC channel model used in simulations

$b$ BER evolution of all methods for time-varying PLC channel

methods, which, in this case, reduce their performance in only $5 \mathrm{~dB}$. This phenomenon occurs primarily due to the high attenuation of the 'worst' channel, which in this case is of about $42 \mathrm{~dB}$ (Fig. 1).

Even when ideal ZF and MMSE are calculated with the same channel used in the transmission to equalise the reception, the progressive performance reduction is caused by the increment of the average channel attenuation and the presence of notches in particular frequencies in which the noise enhancement increases significantly.

Fig. 5 evidences this by illustrating the original and attenuated spectrum of a transmitted signal affected by the channels of Fig. 1. It can be seen that, for the SC-BPSK scheme used in this paper, the spectral-distortion produced by these channels is notorious.

\subsection{BER performance produced by IN}

The purpose of the following simulations is primarily to evaluate the performance of the channel estimation processes when received CS's are distorted not only by the channel frequency response itself, but also by MCA IN. As theoretical ZF and MMSE do not require an estimation algorithm because the channel frequency response is assumed to be known, the following BER will be tested only on CS-based methods.

IN profiles used henceforth are such as the ones shown in Fig. 6, in which AWGN noise profile is shown for comparison purposes. These three different noises are normalised at the same power, and the values of $A$ and $\Gamma$ (Section 3) are also displayed.

The following images represent the BER of transmissions over the three BB-PLC channels previously used, but using IN. Fig. 7 shows two independent simulations performed with two different configuration of MCA over the three PLC channel. For the 'best' channel shown in Fig. 7a, compared with Fig. $4 a$ (the AWGN case), the addition of IN produces an inherent increment in BER regardless the MCA parameters. However, it should be noted the appearance of an error floor on the BER curve for the case of
$A=0.001$ for SNR higher than $18 \mathrm{~dB}$. For the entire SNR range, CS-LS proved to be the most efficient method, followed by CS-MMSE and finally by CS-ZF.

For the 'medium' PLC channel, as shown in Fig. $7 b$, IN produces similar effects to previous simulations, but with an overall performance reduction of about $8 \mathrm{~dB}$. Now, CS-MMSE exceeds CS-LS slightly in both cases. From this simulation, it can be concluded that the CS-ZF technique, while very simple to use, might not be the most appropriate method for applications in PLC. Fig. $7 b$ shows that the best results are achieved by CS-MMSE followed very closely by CS-LS. Nevertheless, it is important to highlight that CS-MMSE demands the variance of the noise and the signal to compute its equalisation term (22) while CS-LS does not (32), making it a much more general, simple and efficient alternative to apply in PLC.

The impact of IN on the 'worst' PLC scenario can be noted in Fig. $7 c$. In this case, compared with the previous simulation, the sharp increase of errors produced by IN is more evident, primarily on the case of $A=0.001$. Here again, CS-MMSE exceeds slightly CS-LS in the range of SNR from 15 to $30 \mathrm{~dB}$, fundamentally in the case of $A=0.001$.

\subsection{BER evolution with time-variant PLC channels}

Real PLC channels varies along the day according to the variation of different impedances (or open circuits) connected to every point of the grid. By using the PLC channels provided by Cañete et al. [18] (Fig. 1), a smooth time-variation between the three channels is simulated and shown in Fig. 8a. This three-dimensional PLC-channel model starts in the 'best' case (time $=0$ ), crosses through the 'medium' case (time $=50)$ and culminates in the 'worst' case $($ time $=100)$, and could be understood as a real PLC-channel variation produced in a period of time (e.g. the evolution of the PLC channel from the morning to the night). 
During these channel variations, a data transmission is performed constantly and the BER of all methods is measured at the receiver and feedbacks to the transmitter (Fig. 2). The noise during these transmissions is AWGN and irrelevant in power, so the effect of the channel variations is what really distorts the received signal and increases BER. The entire procedure follows these rules:

(i) In the case of ideal ZF and MMSE methods, the channel used in both equalisers is the 'best' one and remains fixed during all the time. (ii) CS-based methods begin by identifying the current channel by sending a preamble composed by CS. After that, normally data transmission and subsequent equalisation start until the CS-LS BER exceeds an admissible threshold value of 0.03 .

(iii) When the threshold is exceeded, the receiver feedbacks that to the transmitter which sends a new set of CS with the goal of re-identifying the current channel state and updates the CS-based equalisers.

Fig. $8 b$ shows the BER evolution of all methods against the time-varying channel from Fig. $8 a$. The vertical dotted lines indicate the equaliser updates. The simulation also shows the BER evolution of ideal ZF and MMSE methods in which the channel remains fixed at its initial value. The aim is to show the consequences of time-varying frequency response, and therefore the problem of using detuned or time-fixed equalisers on this type of communication channels.

At the beginning, all methods produce lower, or even null errors, but, due to the differences between the saved equaliser and the continuously changing channel frequency response (CFR), those errors start to rise until the admissible threshold is exceeded. When this happens, CS-based methods update its equaliser taps. As might be expected, after the re-identification process, the BER decreases significantly, and in accordance with the subsequent channel variations, it begins to grow again until the next identification process starts.

\section{Conclusions}

BB-PLCs technique is a promising alternative to deliver information. The main disadvantage of this communication medium is its non-linear and time-varying frequency response, together with the non-Gaussian and high IN environment, which render mandatory the use of some dynamic techniques to reduce BER and offer better transmission speeds. In this paper, all these characteristics were simulated, and furthermore, a proposal of a CP-SC FDE scheme that performs all calculations in the frequency domain is introduced. This algorithm, based on the application of CSs properties, provides an easy way to dynamically compute equalisation coefficients using low computational resources and, in comparison with other alternatives, does not demand the estimation or calculation of signal variances.

Simulations, carried out using three time-fixed PLC channels, INs and, also, a time-varying channel, showed that CSs based methods can even exceed the performance of ideal ZF and MMSE techniques, which use perfect channel knowledge. Also, the measurement of BER when channel identification is compromised by MCA IN proved that channel frequency response estimation can be achieved even for channels with severe attenuations and high-noise levels. For each simulation, the results produced by the ZF technique were worst than MMSE and LS. These two methods showed to work similarly, producing significantly better results than ZF in the case of IN. Comparing these two techniques, the proposal (CS-LS) needs four times less computational resources than CS-MMSE, making it the most interesting technique to apply to real-time PLC systems.

As has been discussed in this paper, PLC channels are constantly changing along the day. By plotting the received error evolution during time-varying channel, this paper emphasises on the importance of a feedback link on PLC systems in order to report growing errors, and therefore update the equaliser taps. Moreover, it has been proved that the use of time-fixed equalisation (or detuned equalisers) is highly not recommended on PLC systems.

\section{Acknowledgments}

This work has been possible thanks to the support granted by the CONICET, ANPCYT, MINCYT, ICYTE, the Universidad Nacional de Mar del Plata and the Universidad Nacional de Misiones.

\section{References}

1 Matanza, J., Alexandres, S., Rodriguez-Morcillo, C.: 'Advanced metering infrastructure performance using European low-voltage power line communication networks', IET Commun., 2014, 8, pp. 1041-1047

2 Corripio, F.J.C., Arrabal, J.A.C., del Rio, L.D., et al.: 'Analysis of the cyclic short-term variation of indoor power line channels', IEEE J. Sel. Areas Commun., 2006, 24, pp. 1327-1338

3 Musolino, A., Raugi, M., Tucci, M.: 'Cyclic short-time varying channel estimation in OFDM power-line communication', IEEE Trans. Power Deliv., 2008, 23, pp. $157-163$

4 Lopes, P.A.C., Pinto, J.M.M., Gerald, J.B.: 'Dealing with unknown impedance and impulsive noise in the power-line communications channel', IEEE Trans. Power Deliv., 2013, 28, pp. 58-66

5 Zimmermann, M., Dostert, K.: 'Analysis and modeling of impulsive noise in broad-band powerline communications', IEEE Trans. Electromagn. Compat. 2002, 44, pp. 249-258

6 Middleton, D.: 'Statistical-physical models of electromagnetic interference', IEEE Trans. Electromagn. Compat., 1977, EMC-19, pp. 106-127

7 Savoia, R., Verde, F.: 'Performance analysis of distributed space-time block coding schemes in Middleton Class-A noise', IEEE Trans. Veh. Technol., 2013, 62, pp. 2579-2595

8 Falconer, D., Ariyavisitakul, S.L., Benyamin-Seeyar, A., et al.: 'Frequency domain equalization for single-carrier broadband wireless systems', IEEE Commun. Mag., 2002, 40, pp. 58-66

9 Yin Hoe, N., Teong Chee, C.: 'Single-carrier cyclic prefix-assisted PLC systems with frequency-domain equalization for high-data-rate transmission', IEEE Trans. Power Deliv., 2010, 25, pp. 1450-1457

10 Benvenuto, N., Tomasin, S.: 'On the comparison between OFDM and single carrie modulation with a DFE using a frequency-domain feedforward filter', IEEE Trans. Commun. , 2002, 50, pp. 947-955

11 Ribeiro, M.V., Colen, G.R., de Campos, F.V.P., et al.: 'Clustered-orthogonal frequency division multiplexing for power line communication: when is it beneficial?', IET Commun., 2014, 8, pp. 2336-2347

12 Morelli, M., Sanguinetti, L., Mengali, U.: 'Channel estimation for adaptive frequency-domain equalization', IEEE Trans. Wirel. Commun., 2005, 4, pp. $2508-2518$

13 Wang, H.M., Gao, X.Q., Jiang, B., et al.: 'Efficient MIMO channel estimation using complementary sequences', IET Commun., 2007, 1, pp. 962-969

14 Wei-Chang, L., Fu-Chun, Y., Ting-Chen, W., et al.: 'A digital Golay-MPIC time domain equalizer for SC/OFDM dual-modes at $60 \mathrm{GHz}$ band', IEEE Trans. Circuits Syst. I, Regul. Pap., 2013, 60, pp. 2730-2739

15 Ming, L., Ye, H.: 'Time-domain channel estimation of high accuracy for LDPC coded SC-FDE system using fixed point decoding in $60 \mathrm{GHz}$ WPAN'. Sixth IEEE Consumer Communications and Networking Conf., 2009. CCNC 2009, 2009 , pp. $1-5$

16 Sobaihi, K., Hammoudeh, A., Scammell, D.: 'FPGA implementation of SC-FDE for $60 \mathrm{GHz}$ WPAN'. 2012 IEEE 23rd Int. Symp. on Personal Indoor and Mobile Radio Communications (PIMRC), 2012, pp. 2009-2014

17 Golay, M.J.E.: 'Complementary series', IRE Trans. Inf. Theory, 1961, 7, pp. 82-87

18 Cañete, F.J., Cortes, J.A., Diez, L., et al.: 'A channel model proposal for indoor power line communications', IEEE Commun. Mag., 2011, 49, pp. 166-174

19 Zimmermann, M., Dostert, K.: 'A multipath model for the powerline channel', IEEE Trans. Commun., 2002, 50, pp. 553-559

20 Develi, I., Kabalci, Y.: 'Highly reliable LDPC coded data transfer in home networks by using Canete's PLC channel model', Int. J. Electr. Power Energy Syst., 2014, 62, pp. 912-918

21 Güzelgöz, S., Arslan, H., Islam, A., et al.: 'A review of wireless and PLC propagation channel characteristics for smart grid environments', J. Electr. Comput. Eng., 2011, Article ID 154040, 12 pages, 2011. doi:10.1155/2011/154040

22 Kim, Y.-H., Song, H.-H., Lee, J.-H., et al.: 'Wideband channel measurements and modeling for in-house power line communication'. Proc. of the Int. Symp. on Power Line Communications and its Applications (ISPLC'02), March 2002

23 Cañete, F.J., Diez, L., Cortes, J.A., et al.: 'Broadband modelling of indoor power-line channels', IEEE Trans. Consum. Electron., 2002, 48, pp. 175-183

24 Meng, H., Guan, Y.L., Chen, S.: 'Modeling and analysis of noise effects on broadband power-line communications', IEEE Trans. Power Deliv., 2005, 20, pp. 630-637

25 Shongwey, T., Vinck, A.J.H., Ferreira, H.C.: 'On impulse noise and its models' 2014 18th IEEE Int. Symp. on Power Line Communications and its Applications (ISPLC), 2014, pp. 12-17

26 Pancaldi, F., Vitetta, G.M., Kalbasi, R., et al.: 'Single-carrier frequency domain equalization', IEEE Signal Process. Mag., 2008, 25, pp. 37-56 